separate protein or a regulatory site must be involved.

The ten chapters on brain and nerve function implicate cyclic AMP as an important regulator of a variety of processes including metabolic activity of the brain, membrane depolarization, transmission at the neuromuscular junction and synaptic functions. There is also an interesting account by Gessa et al. on the behaviourial effects of the dibutyryl analogue of cyclic AMP injected into the brain. As regards analogues of cyclic AMP, there is virtually no discussion of cyclic GMP ; this analogue is known to be metabolically active and has been identified in various tissues. These ten chapters, however, leave no doubt that we shall be hearing a great deal more in the years to come about the importance of cyclic AMP in every aspect of central nervous function, although the key to understanding the role of regulatory molecules is to a large extent dependent on the progress made in the molecular biology of the central nervous system.

Despite some of the omissions that I have pointed out, this volume on cyclic AMP is to be welcomed by all those interested in biological regulatory mechanisms, and the editors and publishers deserve our thanks for its rapid publication. It will be particularly useful to the neurobiologist but may leave some endocrinologists with a nostalgic feeling for the days when the compound was thought to be exclusively designed for hormones to work. This book, which is among the first of many to come in this field, certainly establishes cyclic AMP as an intracellular mediator of diverse metabolic processes of the same class as substances like ATP and coenzyme A.

J. R. TATA

\section{Life Astream}

The Ecology of Running Waters. By H. B. N. Hynes. Pp. xxiv +555 . (Liverpool University: Liverpool, December 1970.) £7.

As the total amount of published work grows, a gap between studies of running water and the rest of limnology will inevitably open and be regretted on all grounds except those of etymology. The word "potamology" will probably come into vogue, though not as a result of any deliberate promotion by $\mathrm{Dr}$ Hynes, who avoids unnecessary long words and jargon. However, this, the first review of the whole field, is bound to provide a base for separate study and a separate name will be demanded.

The first three chapters deal with physical and chemical factors, the next three with attached algae, higher plants and plankton respectively. The text concludes with a chapter on the influence, generally deplorable, of man and, before that, one on primary and secondary production and the path of energy through the ecosystem. Towards the end of the book there are four chapters on fish. The remainder of the text, about half, is devoted to invertebrates. Dr Hynes is a field naturalist with an interest in all organisms that live in running water and a curiosity about what each one does and how they all interact to produce a given type of distribution and a given community structure.

A comparatively small number of groups have invaded fresh water and have adapted their bodies and behaviour to move, respire and feed successfully in a new medium. The first step taken, further modification, has enabled some to occupy specialized niches. The rest struggle in a biotope occupied by many other species. The range of each is determined by a variety of factors, some physical and chemical, others biotic; each sperm must obtain food in competition with others while avoiding becoming food itself. Many insects have had to solve the problem of transition from water to air and back. Few adults appear to be capable of activity at winter air temperatures but, for many larvae, winter is the period of growth, possibly because there is more to eat then or because the species originated in a cold climate, or even because there is less competition or predation at that time. Total production is of academic and practical importance. In no other medium have animals to cope with a constant flow in one direction, with its advantages of a food supply brought to the door and the disadvantages of colonizing a ribbon and regaining ground after a lost foothold.

There is hardly a branch or subbranch of biology into which an incursion has not to be made, but Dr Hynes weaves his material together skilfully to pursue a theme throughout the book. Though it remains clear that his interest is in the living organism in its environment, nothing is allowed to obtrude, and the author maintains an open-minded attitude to classifications of streams and other rigid schemes.

Data from all over the world are discussed. Sometimes the edges of a picture are blurred a little when it is illustrated by different species from different zoogeographical regions. On the other hand, workers in lands where knowledge is less advanced will be grateful that they, as well as Europeans and Americans, can find out from this book what is known.

Hynes produced a book on polluted waters in 1960 but conservationists will find the final chapter of this present one and some other parts essential reading. All concerned with ecology and production biology will find the book of value. If their interest is running water, the book marks a watershed. Any reasonably competent review of this kind would have been welcome; that it is done by an author particularly well qualified is a bonus. Dr Hynes has had practical experience in Europe, North America and Africa. He marshals his facts in an orderly way and presents them in a clear and pleasing style. He has a good knowledge of western European languages. He also has a mother who translates papers in Russian.

T. T. MACAN

\section{Antibiotic Tools}

Nucleoside Antibiotics. By R. J. Suhadolnik. Pp. $\mathrm{x}+442$. (Wiley Interscience: New York and London, January 1971.) $£ 10.75$.

RECENT years have seen a remarkable growth of interest in antibiotics as tools for biochemical research and an associated need that the mode of action should be clearly established. In this context, Dr Suhadolnik's book is a welcome addition to the literature. $\mathrm{He}$ arranges the nucleoside antibiotics in eleven groups. His discussion of each group begins with a short introduction and continues under the headings: discovery, production and isolation; physical and chemical properties ; structure elucidation and chemical synthesis ; biosynthesis ; studies on growth-inhibition; and biochemical properties (usually the most extensive section). The treatment of the material is frankly aimed at understanding modes of action -there is little or no discussion of therapeutic applications. It is pleasing to learn that in several instances detailed studies at the molecular level have been carried out-the elegant work of Ward, Reich and their collaborators on syn/anti conformations of nucleosides, for example, in which the behaviour of formycin-containing polymers played an important part.

The book is not free from defectsthere are numerous examples of proofreading errors, for example, usually not serious. Bacteriological eyebrows may be raised at Salutina lutea (p. 397) and others may ask how the magical properties of heximide (p. 40) managed to escape their attention, but no one is likely to be fooled when told that the addition of the purinyl radical to culture media increases the yield of puromycin from 627 to $479 \mu \mathrm{g} / \mathrm{ml}$. (p. 4). Many of the illustrations seem to have been taken directly, without redrawing, from original publications and occasionally the reproduction is poor.

Puromycin is probably the nucleoside antibiotic with which research workers will be most familiar. One cannot envy anyone the task of disentangling the mode of action of puromycin from current ideas about the basic mechan- 